\title{
Effects of placebo administration on immune mechanisms and relationships with central endogenous opioid
} neurotransmission

\author{
Alan Prossin $\mathbb{D}^{1,2 \times}$, Alisa Koch ${ }^{3}$, Phillip Campbell ${ }^{3}$, Geoffroy Laumet $\mathbb{I D}^{4}$, Christian S. Stohler ${ }^{5}$, Robert Dantzer ${ }^{6}$ and Jon-Kar Zubieta ${ }^{7}$
}

(c) The Author(s) 2021

Behavioral conditioning and expectation can have profound impact on animal and human physiology. Placebo, administered under positive expectation in clinical trials, can have potent effects on disease pathology, obscuring active medications. Emerging evidence suggests placebo-responsive neurotransmitter systems (e.g., endogenous opioid) regulate immune function by manipulating inflammatory proteins including IL-18, a potent pro-inflammatory, nociceptive cytokine implicated in pathophysiology of various diseases. Validation that neuroimmune interactions involving brain $\mu$-opioid receptor (MOR) activity and plasma IL-18 underlie placebo analgesic expectation could have widespread clinical applications. Unfortunately, current lack of mechanistic clarity obfuscates clinical translation. To elucidate neuroimmune interactions underlying placebo analgesia, we exposed 37 healthy human volunteers to a standardized pain challenge on each of 2 days within a Positron Emission Tomography (PET) neuroimaging paradigm using the MOR selective radiotracer, ${ }^{11} \mathrm{C}$-Carfentanil (CFN). Each day volunteers received an intervention (placebo under analgesic expectation or no treatment), completed PET scanning, and rated their pain experience. MOR $\mathrm{BP}_{\mathrm{ND}}$ parametric maps were generated from PET scans using standard methods. Results showed placebo reduced plasma IL-18 during pain $\left(\mathrm{W}_{74}=-3.7, p<0.001\right)$, the extent correlating with reduction in pain scores. Placebo reduction in IL-18 covaried with placebo-induced endogenous opioid release in the left nucleus accumbens $\left(T_{148}=3.33 ; p_{\text {uncorr }}<0.001\right)$ and left amygdala $\left(T_{148}=\right.$ 3.30; $p_{\text {uncorr }}<0.001$ ). These findings are consistent with a modulating effect of placebo (under analgesic expectation in humans) on a potent nociceptive, pro-inflammatory cytokine (IL-18) and underlying relationships with endogenous opioid activity, a neurotransmitter system critically involved in pain, stress, and mood regulation.

Molecular Psychiatry (2022) 27:831-839; https://doi.org/10.1038/s41380-021-01365-x

\section{INTRODUCTION}

Behavioral expectation and conditioning can have profound effects across various medical illnesses including affective disorders [1], irritable bowel syndrome [2], asthma [3], osteoarthritic pain [4], and acute and chronic pain states [5-12]. Administered under positive expectation, placebo can parallel or obscure effects of active medications in clinical trials [13, 14]. However, placebo effects have substantial inter-individual variability potentially linked to their impact on neurotransmitter function (e.g., dopamine, endogenous opioid, endocannabinoid, cholecystokinin) including genetic polymorphisms capable of modulating these neurotransmitter systems [2, 7, 15-17]. Current theories imply that placebo can interact with active medications synergistically, in a disease-modifying fashion [13, 18].

Research highlighting potential disease-modifying effects suggests placebo-responsive neurotransmitter systems can regulate immune function. Peripheral concentrations of interleukin (IL)-1 $\beta$ were selectively linked with endogenous opioid responses in the amygdala in both animal models [19] and humans [20]. Another study showed experimental mood induction regulated plasma IL18, a pro-inflammatory IL-1 family cytokine implicated in the pathophysiology of various medical illnesses [21-28]. Induction of sad mood state increased (and neutral mood reduced) IL-18. Mood-induced regulation of IL-18 covaried with $\mu$-opioid receptor $(\mu O R)$ availability and endogenous opioid release in the nucleus accumbens and amygdala [29-31] during neutral (and sustained sad) affective states. Furthermore, these data suggest bidirectional relationships exist between central neurotransmitters modifiable by placebo administration (e.g., endogenous opioid) and stress-reactive innate immune processes. Table 1.

Studies in both animal models and humans manipulated expectation/conditioning paradigms to investigate placebo's impact on specific immune factors. Pairing a gustatory stimulus (conditioned stimulus/CS) with cyclosporine A (immunosuppressive drug: unconditioned stimulus/US) showed that re-exposure to the CS alone mimicked immunopharmacological properties of

\footnotetext{
${ }^{1}$ Department of Psychiatry and Behavioral Sciences, University of Texas McGovern Medical School, Houston, TX, USA. ${ }^{2}$ Translational Imaging Center, Houston Methodist Research Institute, Weill Cornell College of Medicine, Houston, TX, USA. ${ }^{3}$ Department of Internal Medicine, Division of Rheumatology, University of Michigan Medical School, Ann Arbor, MI, USA. ${ }^{4}$ Department of Physiology, Michigan State University, East Lansing, MI, USA. ${ }^{5}$ College of Dental Medicine, Columbia University, New York, NY, USA. ${ }^{6}$ Department of Symptom Research, Division of Internal Medicine, University of Texas MD Anderson Cancer Center, Houston, TX, USA. ${ }^{7}$ Department of Psychiatry, John T Mather Memorial Hospital, Northwell Health, Port Jefferson, NY, USA. ${ }^{\circledR}$ email: alan.prossin@uth.tmc.edu
}

Received: 8 March 2021 Revised: 5 October 2021 Accepted: 14 October 2021

Published online: 29 October 2021 
Table 1. Pain Measures.

\begin{tabular}{|llll} 
Measure & No Placebo (mean + I- $\mathbf{~ S D )}$ & Placebo (mean $+/-1$ SD) & Wilcoxon W testing (Wilcoxon W, $\boldsymbol{p}$ value) \\
\hline Pain Sensitivity & $1.29+/-1$ & $0.80+/-0.76$ & W74 $=-2.1, p=0.03$ \\
\hline MPQ Sensory Pain & $16+/-7$ & $14+/-8$ & $W 74=-1.5, p>0.10$ \\
\hline MPQ Affective Pain & $2+/-2$ & $1+/-2$ & $W 74=-1.8, p=0.07$
\end{tabular}

Shown in the table are pain measures recorded from the pain challenge and obtained separately for each pre-treatment intervention (e.g., no intervention, placebo intervention). These measures include Pain sensitivity, the average pain intensity measure acquired during the 20 min sustained pain challenge over the volume of hypertonic saline $(\mathrm{ml})$ needed to maintain a constant level of pain over time 47 and the McGill Pain Questionnaire scores which include Sensory and Affective pain scores. All measures were completed following the pain challenge on each of 2 days, the day without placebo pre-treatment and the day with placebo pre-treatment. Here the mean values (+/-1 SD) for each measure are reported in column 2 (no placebo) and column 3 (placebo) of the table. Repeated measures testing (using Wilcoxon W) compared means for each measure on the day of placebo pre-treatment with the respective means on the day with no placebo pre-treatment. Results (Wilcoxon W and un-corrected $p$ value of significance) are reported in column 4 . Significance was set to $p<0.05$ to control for a type 1 error. Results indicated that placebo significantly reduced Pain Sensitivity and MPQ Affective Pain (marginal significance), but not MPQ Sensory Pain scores.

cyclosporine A, evidenced by impaired Th1 cytokine production and decreased $T$ cell proliferation [32, 33]. Repeated pairing of the CS and US, but not single pairings, or simple manipulation of expectations, induced "placebo-elicited" changes in specific inflammatory proteins (e.g., IL-2, IL-6, IL-10, TNF-a) [34-38].

While exact mechanisms remain unclear, evidence suggests central opioid neurotransmitters interact with and potentially regulate peripheral inflammatory processes involving IL-1 family cytokines (e.g., IL-1 $\beta$, IL-18) [20, 29-31]. That IL-18 is further implicated disease pathophysiology [21-28] underscores the clinical translational relevance of these neuroimmune interactions. Here, we examined whether administration of placebo (intravenous isotonic saline in volunteer sight) under expectation of analgesia modulates plasma IL-18. We hypothesized that placebo's immune modulation would be proportional to endogenous opioid activity in brain regions that integrate input from viscera and peripheral nociceptive fibers [39] and immune processes (i.e., central amygdala) [40], modulate outflow to the peripheral immune system (e.g., hypothalamus, amygdala) [30, 41, 42], encode reward expectation and salience (e.g., nucleus accumbens) $[9,43]$, and where we previously identified neuroimmune interactions involving IL-1 family cytokines and $\mu \mathrm{OR}$ activation during negative affective states [20, 29-31].

\section{METHODS}

This research was approved by Institutional Review Boards and Radioactive Drug Research Committees at the University of Michigan and the University of Texas McGovern Medical School. Written informed consent was obtained from all volunteers. The overall research paradigm is depicted in Fig. 1.

\section{Subjects}

A total of 37 right-handed, healthy, non-smoker, male $(n=12)$ and female $(n=25)$ volunteers completed positron emission tomography (PET) brain scans with $\left[{ }^{11} \mathrm{C}\right]$-carfentanil (CFN), a radiotracer with specific binding at the $\mu O R$ [44]. Study volunteers had negative urine drug screens and no medical or psychiatric illness (Structured Clinical Interview for DSM-IV) [45]. Female volunteers reported regular menstrual cycles (28-32 days), negative urine pregnancy testing, and completed PET scanning between day 2 and 10 post menses. No volunteers were using psychotropic/ hormonal medications or compounds with known effects on IL-1 family cytokines or opioid mechanisms for 6 months prior. In prior studies, NEO-PI Neuroticism was associated with immune measures [20], so volunteers completed a personality inventory (NEO PI-R) [46]. Additional measures obtained at study entry (Age, Body Mass Index [BMI: $\left.\mathrm{kg} / \mathrm{m}^{2}\right]$ ) were tested for effects on IL-18.

\section{General study procedure}

Two serial $90 \mathrm{~min}\left[{ }^{11} \mathrm{C}\right]-\mathrm{CFN}$ PET scans were conducted on separate days, with or without placebo treatment as previously described $[7,8]$. The current dataset (acquired between 2007 and 2012) is distinct from the previously described dataset $[7,8]$. Treatment order was randomized and counterbalanced. Placebo treatment involved intravenous injection of 1 $\mathrm{mL}$ of $0.9 \%$ saline in volunteer sight every 4 min during PET scanning, described as "an agent that may increase the body's ability to counter pain". On the day without Placebo, subjects were told they would not receive an analgesic treatment. PET scans began at 1:00 PM (+/- $30 \mathrm{~min})$ and included two 45-minute segments, control (0-45 min) and pain (45-90 $\mathrm{min}$ ) conditions as illustrated in Fig. 1. Prior to each PET scan, two intravenous catheters were placed in antecubital veins for radiotracer administration and blood sampling, permitting venous blood collection in heparinized tubes every $10 \mathrm{~min}$. Upon completion, subjects were informed the "potential pain medication" was placebo.

\section{Experimental pain challenge}

The pain challenge involved a non-painful "control" condition (pain is rated but not delivered) and a sustained pain condition $[8,47]$. The control condition (applied at $0 \mathrm{~min}$ ) involved intramuscular (masseter) injection of non-painful $0.9 \%$ saline over $20 \mathrm{~min}$. The pain condition (applied at $45 \mathrm{~min}$ ) involved intramuscular (masseter) injection of medication-grade $5 \%$ hypertonic saline over $20 \mathrm{~min}$ via a computer-controlled delivery system. The computer recorded subject pain intensity every $15 \mathrm{~s}$ using a visual analog scale (VAS) (0: no pain; 100: most pain imaginable) subsequently altering hypertonic saline delivery to target a $40 \%$ maximum pain intensity as previously described $[48,49]$. Order of conditions (control, pain) was maintained to avoid carry-over effects.

Volume of hypertonic saline required for pain maintenance was recorded, providing an objective measure of Pain Sensitivity for each condition (no treatment, placebo treatment) [48, 49]. Following completion of the challenges, pain was quantified using the McGill Pain Questionnaire (MPQ) [50] yielding MPQ Sensory and Affective Pain scores.

Expectations of placebo analgesia were quantified with a 0-100 VAS scale $(0=$ no expectation, $100=$ expecting complete relief). Subjective assessments of placebo analgesia $(0=$ no relief, $100=$ complete relief $)$ were after placebo scans.

\section{Neuroimaging measures}

Central $\mu \mathrm{OR}$ availability (non-displaceable binding potential, $\mathrm{BP}_{\mathrm{ND}}$ ) was quantified in vivo as described [47]. Scans were acquired using a Siemens $\mathrm{HR}^{+}$scanner (Knoxville, TN) in 3-D mode (FWHM resolution $5.5 \mathrm{~mm}$ inplane and $5.0 \mathrm{~mm}$ axially). Synthesis of ${ }^{11} \mathrm{C}-\mathrm{CFN}$, image acquisition, coregistration, and reconstruction were as previously described $[7,51]$. Maximum mass of CFN was $0.03 \mu \mathrm{g} / \mathrm{kg}$, ensuring sub-pharmacological tracer doses. Injected doses were $15 \pm 1 \mathrm{mCi}(555 \pm 37 \mathrm{MBq}), \quad 50 \%$ administered over $10 \mathrm{~s}$ and $50 \%$ infused over the entire scan to rapidly achieve/maintain steady state. Dynamic images were transformed into two sets of co-registered parametric maps: (a) a tracer transport measure $K_{1}$ ratio) and (b) $\mathrm{BP}_{\mathrm{ND}}$, generated using modified Logan analyses [52] with occipital cortex (devoid of $\mu \mathrm{OR}$ ) as reference region. Approximately 5-7 min after tracer administration, the Logan plot becomes linear with slope proportional to $\mathrm{BP}_{\mathrm{ND}}\left(\mathrm{BP}_{\mathrm{ND}}=\left[\mathrm{B}_{\max } / \mathrm{K}_{\mathrm{d}}\right]+1 ; B_{\max }=\right.$ receptor concentration; $K_{d}=$ receptor affinity). $\mu \mathrm{OR} \mathrm{BP}_{\mathrm{ND}}$ was calculated for control (0-45 min), control + placebo (0-45 min), pain (45-90 min), and pain + placebo (45-90 min) conditions. Structural T1 images, acquired via 3 Tesla MRI scanner (Signa, General Electric, WI) enabled anatomical co-registration and normalization to standardized stereotactic coordinates [47]. 


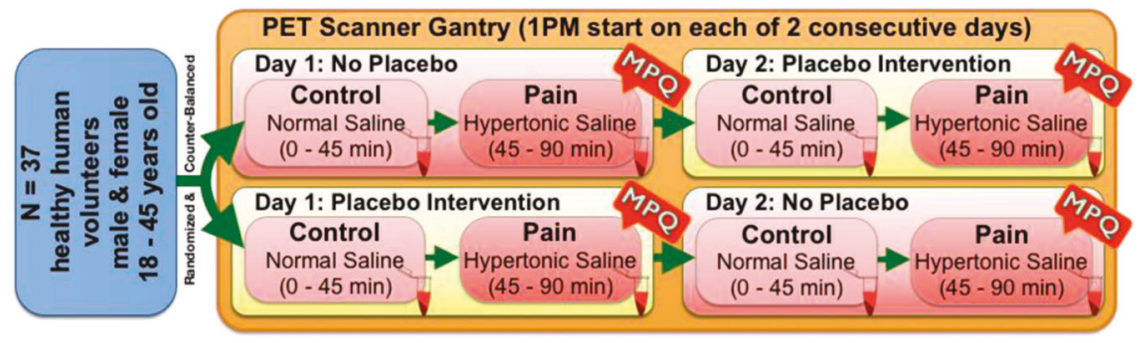

Fig. 1 Research paradigm. The sample derived from $n=37$ healthy volunteers (12 males, 25 females). Once enrolled, volunteers were randomized and counterbalanced to 1 of 2 intervention orders as illustrated by the green double arrows on the left. As such, a given subject was randomized to either Order \#1: No Placebo on Day 1 and Placebo Intervention on day 2 (see top row of diagram) or Order \#2: Placebo intervention on Day 1 and No Placebo on Day 2 (bottom row of diagram). Regarding Placebo/NoPlacebo administration, subjects were told that on one of the two scanning days they will receive an intravenous dose of "an agent that may increase the body's ability to counter pain" and that on the other scanning day they will receive no intervention (e.g., an inert substance). After completion of the studies, the volunteers were informed that the agent was a placebo, an inactive agent.

\section{Measures of inflammation}

Whole blood was obtained from indwelling IV catheter, immediately placed on ice, centrifuged within $30 \mathrm{~min}$ for $15 \mathrm{~min}$ at $4000 \mathrm{rpm}$, separated, and plasma aliquots stored at $-80^{\circ} \mathrm{C}$. Sample storage time averaged $\sim 3-4$ years. Plasma IL-18 concentration was quantified from samples at $45 \mathrm{~min}$ (control condition) and $90 \mathrm{~min}$ (pain condition) for each of treatment condition (placebo, no placebo) via standard ELISA using IL-18 kits (R\&D Systems, MN), samples run in duplicate. To determine assay sensitivity, we assayed serially diluted human IL-18 calibrator. Mean absorbance +2 SD for calibrator diluted to $6.25 \mathrm{pg} / \mathrm{ml}$ was lower than mean absorbance -2SD for calibrator diluted to $12.5 \mathrm{pg} / \mathrm{ml}$ (minimal detectable concentration). Average intra-assay coefficient of variation was calculated to be $3.9 \%$. The highest standard was $1000 \mathrm{pg} / \mathrm{ml}$. IL-18 concentration from each sample was averaged across duplicate pairs.

\section{Planned analyses}

Psychophysical data (Age, BMI, NEO-PI Neuroticism, pain measures) and IL18 were substantially skewed and/or not continuous, violating normality assumptions. These data were analyzed using non-parametric techniques. Mann-Whitney $U$ testing confirmed distributions of psychophysical variables did not differ across sexes. Using IL-18 during the non-painful, control condition we tested both for sex differences in IL-18 (Mann-Whitney $U$ ) and correlations (Spearman) between IL-18 and potential confounders (age, Neuroticism, BMI). Separate Wilcoxon signed rank paired testing showed the effect of pain on plasma IL-18 and the effect of placebo on plasma IL-18. Spearman Rank correlation tested for relationships between pain related variables (changes in IL-18, Pain Sensitivity, MPQ Sensory and Affective scores). Significance for each was set to $p<0.05$ to control for a type 1 error.

\section{Imaging analyses}

involved whole brain voxel-by-voxel ANCOVA testing using SPM12 (Wellcome Trust, England) and MATLAB (Mathworks, MA). Imaging data included control $\mu \mathrm{OR} \mathrm{BP}_{\mathrm{ND}}$ and placebo induced activation of $\mu \mathrm{OR}$ (quantified as change in $\mu \mathrm{OR} \mathrm{BP}_{\mathrm{ND}}$ during control and pain with placebo administration). ANCOVA tested for linear relationships between IL-18 (covariate) and $\mu \mathrm{OR} \mathrm{BP}_{\mathrm{ND}}$ (outcome) during the control condition. Repeated Measures ANCOVA tested for linear relationships between these variables across conditions (i.e., placebo-induced changes during control, pain). Causality isn't inferred from results presented; ANCOVA results are presented as associational only. Based on a-priori hypotheses, we anticipated significant linear relationships between two measures (IL-18, $\mu O R \mathrm{BP}_{\mathrm{ND}}$ ) would occur in a regionally specific manner (amygdala, nucleus accumbens) $[20,29-31,53]$, after correction for multiple comparisons. Significance was set at $p<0.001$ for a priori hypothesized regions and FDRcorrected $p<0.05$ with a 10 -voxel minimum for other regions to control for a type I error.

\section{RESULTS}

Demographic and psychophysical characteristics

The study included 37 healthy humans. Mann-Whitney $U$ testing showed no differences in baseline psychophysical variables (e.g.,
Age, BMI, NEO-PI Neuroticism) between males and females $(P>$ 0.10 for each).

\section{Relationships between $\mu O R \mathrm{BP}_{\mathrm{ND}}$ and IL-18 concentrations during control condition}

No significant relationships were observed between IL-18 and age (rho $=0.21, p>0.10)$ or $\mathrm{BMl}(\mathrm{rho}=0.20, p>0.10)$. There were no sex differences in plasma IL-18 $\left(\mathrm{U}_{37}=0.6 ; p>0.1\right.$; males $(235+/-$ $171 \mathrm{pg} / \mathrm{ml})$, females $(198+/-137 \mathrm{pg} / \mathrm{ml})$. Whole brain, voxel-byvoxel ANCOVA analyses showed plasma IL-18 covaried inversely with $\mu \mathrm{OR} \mathrm{BP}_{\mathrm{ND}}$ during the control condition. Significant effects localized to the left nucleus accumbens $\left(x y z=-11,11,-6 ; T_{35}=\right.$ -2.7 ; $p_{\text {uncorr }}<0.005$; marginally significant) and left amygdala $\left(x y z=-23,-8,-17 ; T_{35}=-3.1 ; p_{\text {uncorr }}<0.001\right)$ (Fig. 2a).

\section{Effects of sustained pain on plasma IL-18}

No sex differences in MPQ Sensory or Affective scores or Pain Sensitivity were observed (Mann-Whiney $U, p>0.10$ for each). Plasma IL-18 was significantly reduced during the pain challenge (Wilcoxon signed rank paired testing $\mathrm{W}_{74}=-2.0, p<0.05$ ) by an average of $12 \mathrm{pg} / \mathrm{ml}$ (6\%) compared to control condition. Paininduced reduction in IL-18 correlated with reduction in MPQ affective pain scores (rho $=0.31, p=0.03$ ), but not with MPQ sensory pain scores (rho $=0.10, p>0.10$ ) or Pain Sensitivity (rho $=$ $0.18, p>0.10)$.

\section{Effect of placebo during control condition}

Placebo significantly reduced plasma IL-18 (Wilcoxon signed rank paired testing, $\mathrm{W}_{74}=-3.5, p<0.001$ : mean IL-18 reduction $97 \mathrm{pg} /$ ml, 46\%) (Fig. 3).

We tested for relationships between placebo-induced changes in plasma IL-18 and endogenous opioid activation (change in $\mu \mathrm{OR}$ $\mathrm{BP}_{\mathrm{ND}}$ from control to control + placebo) using repeated measures ANCOVA. No significant effects were observed between placeboinduced reductions in plasma IL-18 and placebo-induced activation of endogenous opioid neurotransmission during the control conditions $(p>0.001)$.

\section{Effect of placebo during sustained pain challenge}

Wilcoxon signed rank paired testing showed placebo had significant effects, reducing Pain Sensitivity by $17 \%\left(\mathrm{~W}_{74}=-2.1, p=0.03\right)$, marginally reducing MPQ Affective scores, by $18 \%\left(\mathrm{~W}_{74}=-1.8\right.$, $p=0.07)$, but not MPQ Sensory scores $\left(\mathrm{W}_{74}=-1.5, p=0.14\right)$.

Expectations of placebo analgesia correlated with placebo reduction in MPQ Affective pain (rho $=-0.34, p=0.02$ ) and MPQ Sensory pain scores (rho $=-0.30, p=0.03$ ), but not with Pain Sensitivity (rho $=-0.10, p>0.10$ ). Self-reported analgesic effectiveness correlated with placebo reduction in pain sensitivity (rho $=-0.69, p<$ 0.001 ), but not with either MPQ Affective (rho $=-0.16, p>0.10$ ) or MPQ Sensory pain scores (rho $=-0.07, p>0.10$ ). 
a.
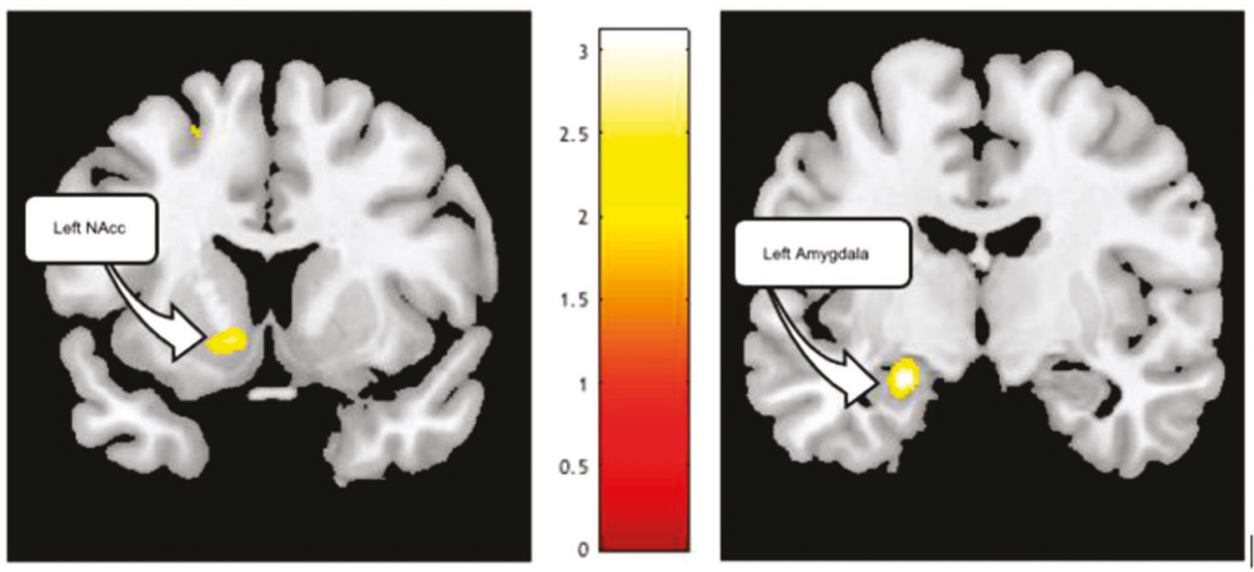

b.
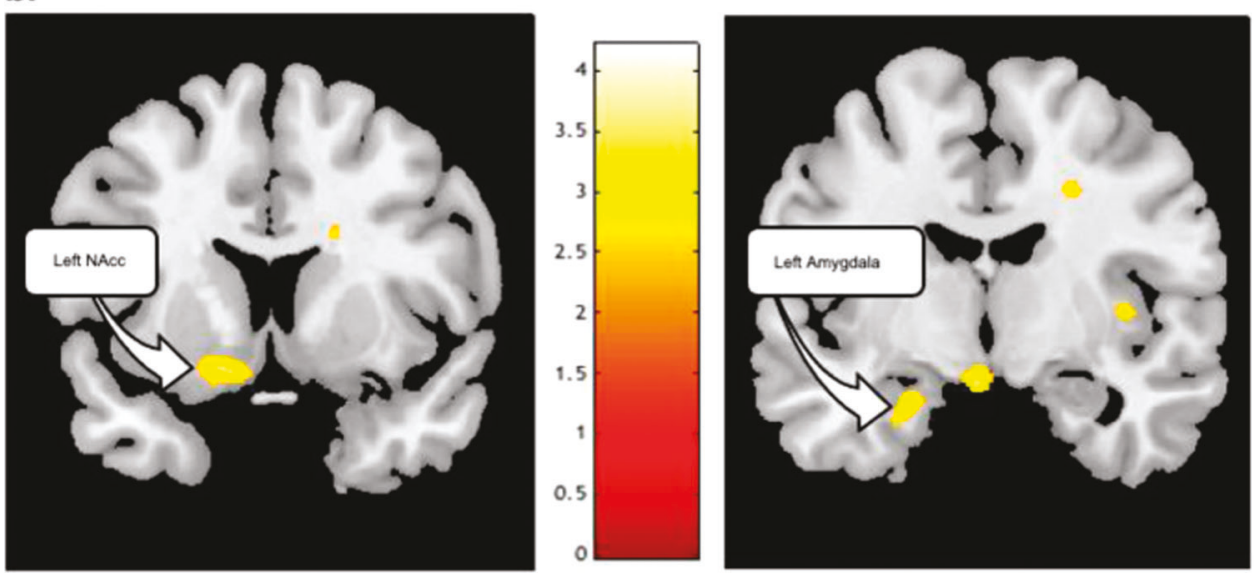

Fig. 2 Relationships between endogenous opioid neurotransmitter activity and plasma IL-18 concentrations during the control condition. In this figure we illustrate these relationships both without placebo intervention (Panel (a) top of Fig. 2) and in response to placebo intervention (Panel (b) bottom of Fig. 2). a Relationship between $\mu$-opioid receptor BPND and plasma concentration of IL-18 during the control condition (e.g., pain anticipation) without placebo administration. The sample derived from $n=37$ healthy volunteers (12 males, 25 females). Significance was set to $p<0.001$ and at least 10 contiguous voxels for regions with a priori regional hypotheses. Correlations reached the threshold for significance in left nucleus accumbens ( $x y z=-11,11,-6 ;$ T35 $=-2.7$; puncorr $<0.005)$ and left amygdala $(x y z=-23,-8,-17$; $\mathrm{T} 35=-3.1$; puncorr $<0.001$ ). Color blobs within brain images represent regional $T$ scores whose values are described in the color bar (inset), ranging from $T=0$ (dark red) to $T>3$ (yellow/white). b Relationship between changes in $\mu$-opioid receptor BPND before and after placebo administration and changes in plasma concentrations of IL-18 during the pain challenge. The sample derived from $n=37$ healthy volunteers (12 males, 25 females). Significance was set to $p<0.001$ and at least 10 contiguous voxels for regions with a priori regional hypotheses. Significant effects were observed in the left nucleus accumbens $(x y z=-12,10,-13 ;$ T148 $=3.33$; puncorr $<0.001)$, and left amygdala $(x y z=$ $-23,-7,-22 ; \mathrm{T} 148=3.30$; puncorr $<0.001$. Color blobs within brain images represent regional $T$ scores whose values are described in the color bar (inset), ranging from $T=0$ (dark red) to $T>3$ (yellow/white).

Significant reductions in IL-18 were observed from pain to pain + placebo conditions $\left(\mathrm{W}_{74}=-3.7, p<0.001\right)$, the extent correlating with placebo-induced reductions in Pain Sensitivity ( $r$ ho $=0.33, p<$ 0.05 ), MPQ Affective pain scores (rho $=0.43, p=0.008$ ), and MPQ Sensory pain scores (rho $=0.33, p<0.05$ ) (Fig. 4). Whole brain, voxelby-voxel repeated measures ANCOVA between plasma IL-18 and endogenous opioid activity showed placebo-induced reductions in IL-18 covaried with placebo-induced endogenous opioid release in the left nucleus accumbens $(x y z=-12,10,-13$; $\left.\mathrm{T}_{148}=3.33 ; \mathrm{p}_{\text {uncorr }}<0.001\right)$ and left amygdala $(\mathrm{xyz}=-23,-7,-22$; $\mathrm{T}_{148}=3.30$; $\mathrm{p}_{\text {uncorr }}<0.001$ ) (Fig. 2b).

\section{DISCUSSION}

We previously showed, in healthy humans during a non-painful, control condition, that plasma IL-1 $\beta$ was inversely proportional to $\mu O R B_{N D}$ in the amygdala bilaterally. Subsequently, sustained pain induced increase in anti-nociceptive IL-1ra covaried with endogenous opioid release in the nucleus accumbens, an effect moderated by pro-nociceptive IL-1 $\beta$, suggesting strong interactions between central endogenous opioid neurotransmitter mechanisms and inflammatory mechanisms [20]. Here we present new data identifying significant neuroimmune interactions between endogenous opioid neurotransmitter activity and another IL-1 family cytokine, IL-18.

IL-18 is a potent pro-nociceptive [54-58], pro-inflammatory, IL-1 family cytokine [59] with morphine tolerance inducing effects $[57,60]$. Plasma IL-18 is regulated in part by binding of endogenous opioid neurotransmitters to surface toll-like receptor-4 on immune cells $[61,62]$. Inactive IL-18 is constitutively expressed in cells throughout the body, many being highly reactive to psychosocial stressors. Inactive "pro-IL-18" remains in the cytosol pending activation, a process involving cleavage of pro-IL-18 via caspase- 1 enzyme. Once activated, IL-18 can readily exit its constituent cell. In anterior pituitary cells, active IL-18 $[63,64]$ readily exits the cell to enter the circulating blood, 
Impact of placebo on plasma IL-18 concentration during the study
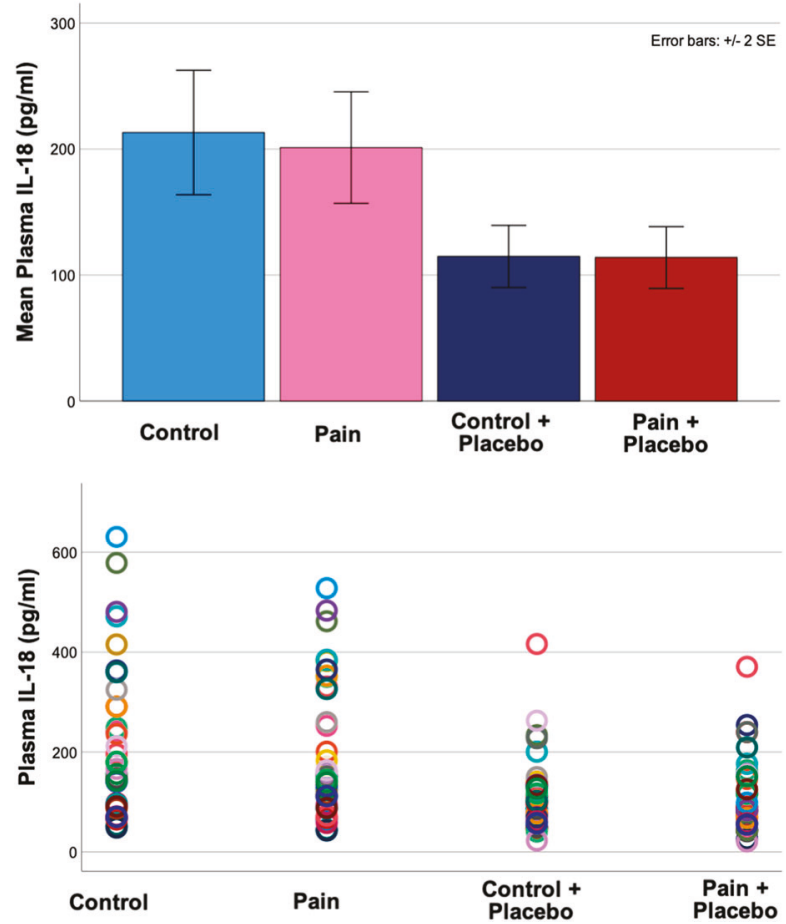

Fig. 3 Impact of placebo on plasma II-18 concentration during the study. The sample comprises $n=37$ healthy volunteers ( 12 males, 25 females). Illustrated in the color bar graphs (top) are mean plasma IL-18 concentrations $(+/-2 \mathrm{SE})$. Colored in blue are mean IL18 concentrations during the control condition without placebo intervention (light blue color) and with placebo intervention (dark blue). Colored in pink/red are mean IL-18 concentrations following sustained pain without placebo intervention (light pink) and with placebo intervention (dark red). Illustrated in the colored dot plots (bottom) are subjects' IL-18 concentrations (vertical axis) separated by experimental condition (Control, Pain, Control + Placebo, Pain + Placebo) on the horizontal axis. Each subject is represented by a specific color. Placebo treatment significantly reduced plasma IL-18 concentration during pain anticipation (W74 $=-3.5, p<0.001$ ), reflecting a mean IL-18 reduction of $97 \mathrm{pg} / \mathrm{ml}$ (46\%) following placebo administration.

providing an explanation for how brain opioids can alter plasma concentration of IL-18. Plasma IL-18 can be modulated by exposure to brief behavioral challenges in both animal models $[65,66]$ and humans [29-31]. In addition, peripheral IL-1 family cytokines can impact brain neurotransmission by an indirect route via activation of vagal afferents which, via neuronal projections to the nucleus of the tractus solitarius and amygdala, can impact stress-reactive neurotransmission [67].

In healthy volunteers resting during the control condition (no pain, no placebo pre-treatment), we showed that plasma IL-18 concentration varied inversely with brain $\mu \mathrm{OR}$ availability (possibly reflecting basal opioid tone [68]) in the left amygdala and left nucleus accumbens. Current understanding of central opioid plasma cytokine neuroimmune interactions suggests it's possible that neuroimmune homeostasis is maintained during relatively nonstressful events (e.g., control condition) in part via bi-directional interactions between neuronal processes (e.g., endogenous opioid tone in the Amygdala) and peripheral inflammatory protein activation (plasma IL-18), which vary linearly in our sample during the restful control condition. Future studies quantifying vagal afferent activity may better elucidate these bi-directional relationships.

During the pain challenge we showed a small but significant reduction in plasma IL-18 which covaried with MPQ affective pain but not MPQ sensory pain scores. This was unexpected as we anticipated pain-induced plasma IL-18 elevation. However, in retrospect, given that study subjects are otherwise healthy, without acute or chronic pain, and for whom resolution of pain occurred shortly after the challenge, it is possible that physiological mechanisms in these healthy subjects were not overwhelmed during the experimental challenge. Furthermore, these potential physiological mechanisms appear adequately functioning, reducing a potent nociceptive protein (IL-18) and potentially reducing persistence of pain in these healthy individuals. Evidence from animal models suggests that under physiological conditions and in response to certain stressors, effects of IL-18 are buffered by another potentially stress-reactive, endogenous protein, IL-18 binding protein (IL-18bp) [69]. While we have not quantified IL$18 \mathrm{bp}$ in the current study, we identified a reduction in IL-18, suggesting there was a concurrent increase in IL-18bp, buffering the potent pain inducing effects of IL-18.

We showed that administration of placebo (with expectation of analgesia) significantly reduced plasma concentration of IL-18 by $46 \%$ during the control condition. Indeed, IL-18 is implicated in emergence of hyperalgesia [70, 71] and morphine tolerance [60]. We also showed extent of subjects' expectation of placebo analgesia (quantified prior to pain challenge) significantly correlated with MPQ Affective and Sensory pain scores. Taken together, these findings suggest expectation of placebo analgesia, via reduction in pronociceptive IL-1 family cytokines (e.g., IL-18) during the control condition, may set the stage for reduction in MPQ pain scores quantified during the pain challenge.

Placebo significantly reduced plasma IL-18 during the pain condition. The magnitude of IL-18 reduction correlated with the magnitude of reduction in MPQ Affective and Sensory pain scores and an objective measure, Pain Sensitivity. Furthermore, placeboinduced reduction in IL-18 covaried with placebo induced endogenous opioid release in the left nucleus accumbens and left amygdala. As noted in a review by Vits and colleagues [36], A prerequisite for the classical conditioning of immune functions is the functional interaction between the central nervous system (CNS) and the peripheral immune system [72-74]. In their review, Vits and colleagues list the autonomic nervous system as a key pathway for communication between the Central Nervous System (CNS) and the peripheral immune system during conditioned immunosuppression. However, despite this and other encouraging research [75], the role of CNS neurotransmitters (and brain regional localization) in behavioral conditioning of immune function (and in immune modulation during human placebo expectation) remain incompletely understood. To our knowledge, this is the first in-vivo human study to identify mind-body interactions potentially underlying potent analgesic effects of placebo. Here, during the control condition, whole brain, voxel by voxel ANCOVA showed placebo-induced change in plasma IL-18 did not covary with placebo-induced change in $\mu \mathrm{OR}$ availability. In contrast, during the pain challenge, prominent covariant effects were identified between placebo-induced reduction in plasma IL-18 and placeboinduced endogenous opioid release, interactions during a more stressful, painful state.

Note that placebo-induced neuroimmune interactions identified during the pain condition localized to the Amygdala and Nucleus Accumbens, the same brain regions wherein we previously identified interactions between plasma IL-18 and brain endogenous opioid release during induction of a negative affective state. This finding is consistent with our original hypothesis that magnitude of placebo's immune modulating effects are proportional to endogenous opioid release within brain regions that integrate input from viscera and peripheral nociceptive fibers [39] and immune processes [40] (central amygdala), modulate outflow to the peripheral immune system (hypothalamus, amygdala) [48, 59-62] and encode reward expectation and salience (nucleus accumbens) [9, 43]. However, mechanisms underlying lateralization of imaging findings (and clinical impact 

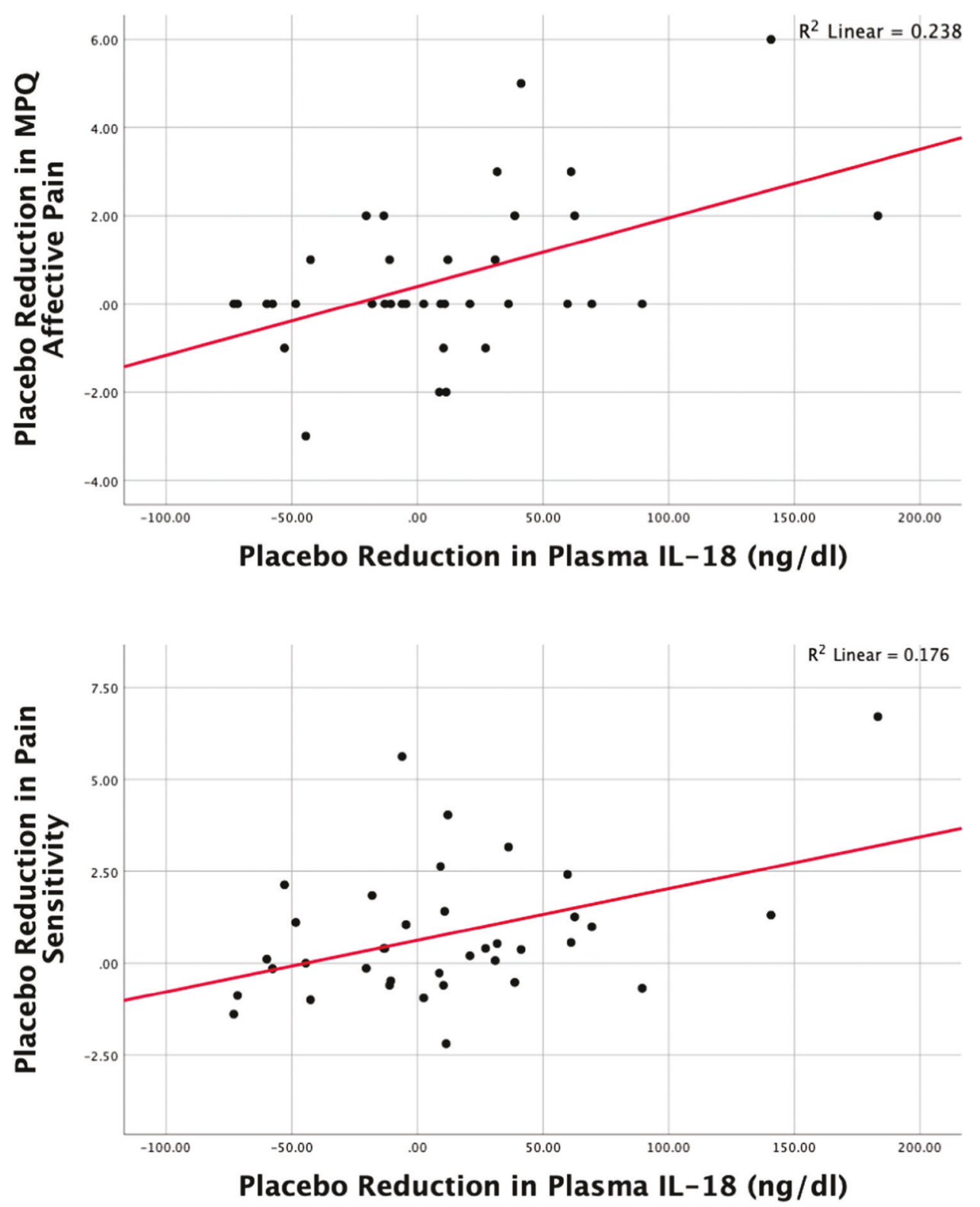

Fig. 4 Relationships between placebo-induced changes in MPQ affective pain (top graph) and pain sensitivity scores (bottom graph) with placebo induced changes in plasma IL-18 are depicted in this graph. The sample derived from $n=37$ healthy volunteers (12 males, 25 females). (Note: correlation testing with placebo-induced changes in MPQ Sensory Pain score was not completed given the lack of significance in testing of correlations between MPQ Sensory Pain and pain induced change in plasma IL-18). The top graph shows the correlation between placebo-induced reductions in MPQ Affective pain scores and placebo-induced changes in IL-18 plasma levels during the pain challenge $(r=$ $-0.49, p=0.001)$. The bottom graph shows similar relationships for placebo induced changes in Pain Sensitivity and IL-18 during pain ( $r=$ $-0.42, p<0.005)$.

on pain) are unclear. Strict lateralization of brain effects was not present in our prior studies of placebo-induced opioidergic activity $[7,8]$ or neuroimmune interactions during experimental mood induction [29-31]. Future studies specifically probing factors underlying lateralization effects would help clarify these issues.

While the results presented are novel and provide evidence supporting development of innovative, personalized expectationbased interventions, certain factors limit the extent of translation to treatment of clinical pain states. Subjects included in the current study were healthy, without acute/chronic medical illness including pain, so our finding may reflect normal physiologic processes. That these findings were present in the absence of disease further underscores their importance in informing understanding of currently unknown, mechanisms potentially underlying placebo effect in general and placebo analgesia in particular. However, the underlying study design isn't optimal to inform on functional relevance. Expansion of investigation in patient populations (within a paradigm developed to inform functional relevance) would help elucidate functional translation of our findings to disease pathology. Additionally, given data skewing, we used non-parametric methods. We recognize that, in presence of normally distributed data and adequate sample size, implementing ANOVA/ANCOVA analyses with a $2 \times 2$ factorial design could permit more robust interaction analyses involving covariates. Finally, while our experimental strategy created a condition of placebo expectation, we didn't control for effect (without expectation) of so-called vehicle. In contrast to the placebo condition where normal saline was injected in full view of the 
subjects (described as an injection of analgesic) to elicit expectation of placebo analgesia, during the control condition, no injection was given (in full view of subjects) to limit subjects' expectation of analgesia. Control for the vehicle via a third condition wherein normal saline was injected and described as an inert substance with no related analgesia may further isolate effects related to expectation. Other strategies employing patient blinding are worthy of consideration for future studies. In addition, variation in sample storage time could impact inter-individual comparisons. However, all samples for each subject were stored for the same duration. Given our primary focus on testing within subject effects, we don't expect inter-subject variation in sample storage confounded these findings.

\section{REFERENCES}

1. Kirsch I. Placebo effect in the treatment of depression and anxiety. Front Psychiatry. 2019;10:407.

2. Hall KT, Lembo AJ, Kirsch I, Ziogas DC, Douaiher J, Jensen KB, et al. Catechol-Omethyltransferase val158met polymorphism predicts placebo effect in irritable bowel syndrome. PLoS ONE. 2012;7:e48135.

3. Wechsler ME, Kelley JM, Boyd IO, Dutile S, Marigowda G, Kirsch I, et al. Active albuterol or placebo, sham acupuncture, or no intervention in asthma. N. Engl J Med. 2011;365:119-26.

4. Jacobson AF, Umberger WA, Palmieri PA, Alexander TS, Myerscough RP, Draucker $\mathrm{CB}$, et al. Guided imagery for total knee replacement: a randomized, placebocontrolled pilot study. J Alter Complement Med. 2016;22:563-75.

5. Jensen KB, Kaptchuk TJ, Kirsch I, Raicek J, Lindstrom KM, Berna C, et al. Nonconscious activation of placebo and nocebo pain responses. Proc Natl Acad Sci USA. 2012;109:15959-64.

6. Harris RE, Zubieta JK, Scott DJ, Napadow V, Gracely RH, Clauw DJ. Traditional Chinese acupuncture and placebo (sham) acupuncture are differentiated by their effects on mu-opioid receptors (MORs). Neuroimage. 2009;47:1077-85.

7. Scott DJ, Stohler CS, Egnatuk CM, Wang H, Koeppe RA, Zubieta JK. Placebo and nocebo effects are defined by opposite opioid and dopaminergic responses. Arch Gen Psychiatry. 2008;65:220-31.

8. Zubieta JK, Bueller JA, Jackson LR, Scott DJ, Xu Y, Koeppe RA, et al. Placebo effects mediated by endogenous opioid activity on mu-opioid receptors. J Neurosci. 2005;25:7754-62.

9. Zubieta JK, Stohler CS. Neurobiological mechanisms of placebo responses. Ann N Y Acad Sci. 2009;1156:198-210.

10. Wager TD, Scott DJ, Zubieta JK. Placebo effects on human mu-opioid activity during pain. Proc Natl Acad Sci USA. 2007;104:11056-61.

11. Carvalho C, Caetano JM, Cunha L, Rebouta P, Kaptchuk TJ, Kirsch I. Open-label placebo treatment in chronic low back pain: a randomized controlled trial. Pain. 2016;157:2766-72.

12. Kleine-Borgmann J, Schmidt K, Hellmann A, Bingel U. Effects of open-label placebo on pain, functional disability, and spine mobility in patients with chronic back pain: a randomized controlled trial. Pain. 2019;160:2891-7.

13. Enck $P$, Klosterhalfen $S$. The placebo response in clinical trials-the current state of play. Complement Ther Med. 2013;21:98-101.

14. Kirsch I. The clinical significance of drug-placebo differences. Epidemiol Psychiatr Sci. 2018;27:240-1.

15. Pecina M, Love T, Stohler CS, Goldman D, Zubieta JK. Effects of the Mu opioid receptor polymorphism (OPRM1 A118G) on pain regulation, placebo effects and associated personality trait measures. Neuropsychopharmacology. 2015;40:957-65.

16. Benedetti F. Placebo analgesia. Neurol Sci. 2006;27:S100-2.

17. Colloca L, Wang Y, Martinez PE, Chang YC, Ryan KA, Hodgkinson C, et al. OPRM1 rs1799971, COMT rs4680, and FAAH rs324420 genes interact with placebo procedures to induce hypoalgesia. Pain. 2019;160:1824-34.

18. Evers AWM, Colloca L, Blease C, Annoni M, Atlas LY, Benedetti F, et al. Implications of placebo and nocebo effects for clinical practice: expert consensus. Psychother Psychosom. 2018;87:204-10.

19. Day HE, Curran EJ, Watson SJ Jr, Akil H. Distinct neurochemical populations in the rat central nucleus of the amygdala and bed nucleus of the stria terminalis: evidence for their selective activation by interleukin-1beta. J Comp Neurol. 1999;413:113-28.

20. Prossin AR, Zalcman SS, Heitzeg MM, Koch $A E$, Campbell $P L$, Phan $K L$, et al Dynamic interactions between plasma IL-1 family cytokines and central endogenous opioid neurotransmitter function in humans. Neuropsychopharmacology. 2015;40:554-65.

21. Cho SX, Berger PJ, Nold-Petry CA, Nold MF. The immunological landscape in necrotising enterocolitis. Expert Rev Mol Med. 2016;18:e12.
22. Malhotra R, Siew ED. Biomarkers for the early detection and prognosis of acute kidney injury. Clin J Am Soc Nephrol. 2017;12:149-73.

23. Liu RW, Du JT, Liu YF, Liu SX. [Expression and role of IL-18 in chronic rhinosinusitis]. Lin Chung Er Bi Yan Hou Tou Jing Wai Ke Za Zhi. 2018;32:497-501.

24. Liu D, Zeng X, Li X, Mehta JL, Wang X. Role of NLRP3 inflammasome in the pathogenesis of cardiovascular diseases. Basic Res Cardiol. 2018;113:5.

25. Liu S, Liu YP, Lv Y, Yao JL, Yue DM, Zhang MY, et al. IL-18 contributes to bone cancer pain by regulating glia cells and neuron interaction. J Pain. 2018;19:186-95.

26. Cao Q, Cai W, Niu G, He L, Chen X. Multimodality imaging of IL-18-binding protein-Fc therapy of experimental lung metastasis. Clin Cancer Res. 2008;14:6137-45.

27. Gunel N, Coskun U, Sancak B, Hasdemir O, Sare M, Bayram O, et al. Prognostic value of serum IL-18 and nitric oxide activity in breast cancer patients at operable stage. Am J Clin Oncol. 2003;26:416-21.

28. Li LL, Deng XF, Li JP, Ning N, Hou XL, Chen JL. Association of IL-18 polymorphisms with rheumatoid arthritis: a meta-analysis. Genet Mol Res. 2016;15:1-15.

29. Prossin AR, Koch AE, Campbell PL, Barichello T, Zalcman SS, Zubieta JK. Experimental sadness induces relevant interactions between central endogenous opioid activation and plasma IL-18 concentrations in depressed volunteers. Mol Psychiatry. 2016;21:151.

30. Prossin AR, Koch AE, Campbell PL, Barichello T, Zalcman SS, Zubieta JK. Acute experimental changes in mood state regulate immune function in relation to central opioid neurotransmission: a model of human CNS-peripheral inflammatory interaction. Mol Psychiatry. 2016;21:243-51.

31. Prossin AR, Koch AE, Campbell PL, Mclnnis MG, Zalcman SS, Zubieta JK. Association of plasma interleukin-18 levels with emotion regulation and mu-opioid neurotransmitter function in major depression and healthy volunteers. Biol Psychiatry. 2011;69:808-12.

32. Goebel MU, Trebst AE, Steiner J, Xie YF, Exton MS, Frede S, et al. Behavioral conditioning of immunosuppression is possible in humans. FASEB J. 2002;16:1869-73.

33. Wirth T, Ober K, Prager G, Vogelsang M, Benson S, Witzke O, et al. Repeated recall of learned immunosuppression: evidence from rats and men. Brain Behav Immun. 2011;25:1444-51.

34. Albring A, Wendt L, Benson S, Witzke O, Kribben A, Engler H, et al. Placebo effects on the immune response in humans: the role of learning and expectation. PLoS ONE. 2012;7:e49477.

35. Grigoleit JS, Kullmann JS, Winkelhaus A, Engler H, Wegner A, Hammes F, et al. Single-trial conditioning in a human taste-endotoxin paradigm induces conditioned odor aversion but not cytokine responses. Brain Behav Immun. 2012;26:234-8

36. Vits S, Cesko E, Enck P, Hillen U, Schadendorf D, Schedlowski M. Behavioural conditioning as the mediator of placebo responses in the immune system. Philos Trans R Soc Lond B Biol Sci. 2011;366:1799-807.

37. Schedlowski M, Pacheco-Lopez G. The learned immune response: pavlov and beyond. Brain Behav Immun. 2010;24:176-85.

38. Pacheco-Lopez G, Engler H, Niemi MB, Schedlowski M. Expectations and associations that heal: Immunomodulatory placebo effects and its neurobiology. Brain Behav Immun. 2006:20:430-46.

39. Neugebauer V, Li W, Bird GC, Han JS. The amygdala and persistent pain. Neuroscientist. 2004;10:221-34.

40. Thinschmidt JS, King MA, Korah M, Perez PD, Febo M, Miyan J, et al. Central neural activation following contact sensitivity peripheral immune challenge: evidence of brain-immune regulation through $C$ fibres. Immunology. 2015;146:206-16.

41. Pacheco-Lopez G, Niemi MB, Kou W, Harting M, Fandrey J, Schedlowski M. Neural substrates for behaviorally conditioned immunosuppression in the rat. J Neurosci. 2005;25:2330-7.

42. Tsigos C, Chrousos GP. Hypothalamic-pituitary-adrenal axis, neuroendocrine factors and stress. J Psychosom Res. 2002;53:865-71.

43. Haber SN, McFarland NR. The concept of the ventral striatum in nonhuman primates. Ann N.Y Acad Sci. 1999;877:33-48.

44. Dannals RF, Ravert HT, Frost JJ, Wilson AA, Burns HD, Wagner HN Jr. Radiosynthesis of an opiate receptor binding radiotracer: [11C]carfentanil. Int J Appl Radiat Isot. 1985;36:303-6.

45. First MB, Spitzer R. Structured Clinical Interview for DSM-IV Axis I Disorders (SCID-I). 1995.

46. Costa PT, McCrae RR. Revised NEO Personality Inventory and Five-Factor Inventory professional manual. Psychological Assessment Resources.: Odessa, FL., 1992.

47. Zubieta JK, Smith YR, Bueller JA, Xu Y, Kilbourn MR, Jewett DM, et al. Regional mu opioid receptor regulation of sensory and affective dimensions of pain. Science. 2001;293:311-5.

48. Stohler CS, Kowalski CJ. Spatial and temporal summation of sensory and affective dimensions of deep somatic pain. Pain. 1999;79:165-73. 
49. Zhang X, Ashton-Miller JA, Stohler CS. A closed-loop system for maintaining constant experimental muscle pain in man. IEEE Trans Biomed Eng. 1993;40:344-52.

50. Melzack R. The McGill Pain Questionnaire: major properties and scoring methods. Pain. 1975;1:277-99.

51. Zubieta JK, Heitzeg MM, Smith YR, Bueller JA, Xu K, Xu Y, et al. COMT val158met genotype affects mu-opioid neurotransmitter responses to a pain stressor. Science. 2003;299:1240-3.

52. Logan J, Fowler JS, Volkow ND, Wang GJ, Ding YS, Alexoff DL. Distribution volume ratios without blood sampling from graphical analysis of PET data. J Cereb Blood Flow Metab. 1996:16:834-40.

53. Prossin AR, Love TM, Koeppe RA, Zubieta JK, Silk KR. Dysregulation of regional endogenous opioid function in borderline personality disorder. Am J Psychiatry. 2010;167:925-33.

54. Jurga AM, Piotrowska A, Makuch W, Przewlocka B, Mika J. Blockade of P2X4 receptors inhibits neuropathic pain-related behavior by preventing MMP-9 activation and, consequently, pronociceptive interleukin release in a Rat Model. Front Pharm. 2017;8:48.

55. Li SS, Zhang WS, Ji D, Zhou YL, Li H, Yang JL, et al. Involvement of spinal microglia and interleukin-18 in the anti-nociceptive effect of dexmedetomidine in rats subjected to CCI. Neurosci Lett. 2014;560:21-5.

56. Li WW, Guo TZ, Liang D, Shi X, Wei T, Kingery WS, et al. The NALP1 inflammasome controls cytokine production and nociception in a rat fracture model of complex regional pain syndrome. Pain. 2009;147:277-86.

57. Pilat D, Piotrowska A, Rojewska E, Jurga A, Slusarczyk J, Makuch W, et al. Blockade of IL-18 signaling diminished neuropathic pain and enhanced the efficacy of morphine and buprenorphine. Mol Cell Neurosci. 2016;71:114-24.

58. Verri WA Jr., Cunha TM, Magro DA, Domingues AC, Vieira SM, Souza GR, et al. Role of IL-18 in overt pain-like behaviour in mice. Eur J Pharmacol. 2008;588:207-12.

59. Dinarello CA, Novick D, Kim S, Kaplanski G. Interleukin-18 and IL-18 binding protein. Front Immunol. 2013;4:289.

60. Chen ML, Cao H, Chu YX, Cheng LZ, Liang LL, Zhang YQ, et al. Role of P2X7 receptor-mediated IL-18/L-18R signaling in morphine tolerance: multiple glialneuronal dialogues in the rat spinal cord. J Pain. 2012;13:945-58.

61. Jurga AM, Rojewska E, Makuch W, Mika J. Lipopolysaccharide from Rhodobacter sphaeroides (TLR4 antagonist) attenuates hypersensitivity and modulates nociceptive factors. Pharm Biol. 2018;56:275-86.

62. Popiolek-Barczyk K, Piotrowska A, Makuch W, Mika J. Biphalin, a Dimeric Enkephalin, Alleviates LPS-Induced activation in rat primary microglial cultures in opioid receptor-dependent and receptor-independent manners. Neural Plast. 2017;2017:3829472.

63. Wang N, Sugama S, Conti B, Teramoto A, Shibasaki T. Interleukin-18 mRNA expression in the rat pituitary gland. J Neuroimmunol. 2006;173:117-25.

64. Nagai $Y$, Ogasawara $H$, Taketa $Y$, Aso H, Kanaya T, Miyake M, et al. Expression of inflammatory-related factors in porcine anterior pituitary-derived cell line. Vet Immunol Immunopathol. 2008:124:201-8.

65. Conti B, Sugama S, Kim Y, Tinti C, Kim H, Baker H, et al. Modulation of IL-18 production in the adrenal cortex following acute ACTH or chronic corticosterone treatment. Neuroimmunomodulation. 2000;8:1-7.

66. Sugama S, Conti B. Interleukin-18 and stress. Brain Res Rev. 2008;58:85-95.

67. Day HE, Akil H. Differential pattern of c-fos mRNA in rat brain following central and systemic administration of interleukin-1-beta: implications for mechanism of action. Neuroendocrinology. 1996;63:207-18.

68. Smith YR, Stohler CS, Nichols TE, Bueller JA, Koeppe RA, Zubieta JK. Pronociceptive and antinociceptive effects of estradiol through endogenous opioid neurotransmission in women. J Neurosci. 2006;26:5777-85.

69. Dinarello CA. Targeting interleukin 18 with interleukin 18 binding protein. Ann Rheum Dis. 2000;59:117-20.

70. Cordero MD, Alcocer-Gomez E, Culic O, Carrion AM, de Miguel M, Diaz-Parrado E, et al. NLRP3 inflammasome is activated in fibromyalgia: the effect of coenzyme Q10. Antioxid Redox Signal. 2014;20:1169-80.

71. Vasudeva K, Vodovotz Y, Azhar N, Barclay D, Janjic JM, Pollock JA. In vivo and systems biology studies implicate IL-18 as a central mediator in chronic pain. J Neuroimmunol. 2015;283:43-9.

72. Dantzer R, O'Connor JC, Freund GG, Johnson RW, Kelley KW. From inflammation to sickness and depression: when the immune system subjugates the brain. Nat Rev Neurosci. 2008;9:46-56.

73. Glaser R, Kiecolt-Glaser J. How stress damages immune system and health. Disco Med. 2005;5:165-9.

74. Tracey KJ. Reflex control of immunity. Nat Rev Immunol. 2009;9:418-28.

75. Ben-Shaanan TL, Azulay-Debby H, Dubovik T, Starosvetsky E, Korin B, Schiller M, et al. Activation of the reward system boosts innate and adaptive immunity. Nat Med. 2016:22:940-4.

\section{ACKNOWLEDGEMENTS}

The authors would like to acknowledge the contributions of the late Steven Zalcman, PhD, Department of Psychiatry, University of Medicine and Dentistry of New Jersey, Newark, NJ who provided psychoneuroimmunology guidance and mentoring in the planning stages of the project. Dr. Stacia DeSantis provided statistical guidance during the revision of the manuscript. Additional contributions to this research are mentioned below. Zubieta Lab: Virginia Murphy-Weinberg, Tiffany Love, Heng Wang, Marta Pecina, MBNI staff. Nuclear Medicine: Jill Rothley, Edward McKenna, Andrew Weeden, Paul Kison, Shayna Huber.

\section{AUTHOR CONTRIBUTIONS}

AP conceptualized the basic premise of the study, investigating the impact of placebo on specific opioid-cytokine interactions and relation to placebo analgesia. As such, he made critical contributions to the conceptualization and implementation of neuroimmune aspects of the study research paradigm and analyses. He assisted Mr. Campbell in completing ELISA assays. He developed (and edited) the first and subsequent drafts of the paper, edited the final draft, and developed (and edited) the response to reviewers. He provided (together with Zubieta lab staff) data curation. He developed the statistical design and completed statistical analyses for the study. AK provided expert guidance on inflammatory aspects of the study. Based on her experience in the rheumatology lab with animal models, she provided suggestions as to benefits of focusing inflammatory aspects of the study on interleukin-18. She provided reviews/edits of paper drafts. PC supervised activities in the Koch Lab. He obtained all ELISA kits from the manufacturer and led (with assistance from AP) efforts to quantify IL-18 via standard ELISA assays, using manufacturer provided ELISA kits, and following manufacturer instructions. PC assisted with validation and review of IL-18 data in the current study. He was provided an opportunity to contribute to paper drafts. GL provided expert input on the underlying role of IL-1 family cytokines in the emergence and persistence of pain. He contributed to interpretation of results, paper drafts, and reviews/edits. CS (together with JKZ) conceptualized the overall pain challenge which they implemented in several studies. He provided edits of the paper drafts and response to reviewers. RD provided expert input on experimental planning and interpretation of results. He provided critical feedback in interpreting results and provided input on paper drafts and reviews/edits. JKZ made critical contributions to conceptualization and research design of pain and opioid imaging aspects of the study and contributed to design of neuroimmune aspects of the study. He reviewed/edited statistical design of the project and presentation of the data. He contributed to review and edit of the paper drafts and response to reviewers.

\section{FUNDING}

Research Support to AP came from the University of Michigan Comprehensive Depression Center Rachel Upjohn Clinical Scholars Award and the National Institutes of Health (K99/R00 DA033454, R61 NS113316). Research support to JKZ came from the National Institutes of Health (R01 DA016423, R01 DA022520) and the Phil F. Jenkins Foundation. Research support to AK came from The Frederick G.L. Huetwell and William D. Robinson Professorship at the University of Michigan, the Veterans Administration Research Service, and the National Institutes of Health (R01 AR48267). Research support to Robert Dantzer came from the National Institutes of Health (R01 CA193522, R01 NS073939) and from a National Institutes of Health Cancer Center Support (CORE) grant (P30 CA016672 to the University of Texas MD Anderson).

\section{COMPETING INTERESTS}

Dr. Koch has consulted for Metastatix, Pennside Partners, Cypress Bioscience, Takeda Pharmaceuticals, NiCox SA, and Celtaxsys and has been an expert for Kirkland and Ellis. Dr. Koch was a consultant for Gerson Lehrman Group of Healthcare and Biomedical Advisors, Guidepoint Global, UCB Pharmaceuticals, and the Fund for Autoimmune Research. Dr. Koch has received investigator-initiated research grants for Bristol-Myers Squibb, Roche, and Takeda Pharmaceuticals. Dr. Koch is currently employed by Eli Lilly and Company. All other authors report no biomedical financial or potential conflicts of interest.

\section{ADDITIONAL INFORMATION}

Correspondence and requests for materials should be addressed to Alan Prossin.

Reprints and permission information is available at http://www.nature.com/ reprints

Publisher's note Springer Nature remains neutral with regard to jurisdictional claims in published maps and institutional affiliations. 
Open Access This article is licensed under a Creative Commons Ac) Attribution 4.0 International License, which permits use, sharing,
adaptation, distribution and reproduction in any medium or format, as long as you give appropriate credit to the original author(s) and the source, provide a link to the Creative Commons license, and indicate if changes were made. The images or other third party material in this article are included in the article's Creative Commons license, unless indicated otherwise in a credit line to the material. If material is not included in the article's Creative Commons license and your intended use is not permitted by statutory regulation or exceeds the permitted use, you will need to obtain permission directly from the copyright holder. To view a copy of this license, visit http://creativecommons. org/licenses/by/4.0/.

(c) The Author(s) 2021 